\title{
Optimal Wind Bidding Strategies in Day-Ahead Markets
}

\author{
Isaias L.R. Gomes ${ }^{2}$, Hugo M.I. Pousinho ${ }^{1}$, Rui Melício ${ }^{1,2(\bowtie)}$, \\ and Victor M.F. Mendes 2,3 \\ ${ }^{1}$ IDMEC/LAETA, Instituto Superior Técnico, \\ Universidade de Lisboa, Lisbon, Portugal \\ ruimelicio@gmail.com \\ 2 Departamento de Física, Escola de Ciências \\ e Tecnologia Universidade de Évora, Évora, Portugal \\ ${ }^{3}$ Instituto Superior de Engenharia de Lisboa, Lisbon, Portugal
}

\begin{abstract}
This paper presents a computer application (CoA) for wind energy (WEn) bidding strategies (BStr) in a pool-based electricity market (EMar) to better accommodate the variability of the renewable energy (ReEn) source. The $\mathrm{CoA}$ is based in a stochastic linear mathematical programming (SLPr) problem. The goal is to obtain the optimal wind bidding strategy (OWBS) so as to maximize the revenue (MRev). Electricity prices (EPr) and financial penalties (FiPen) for shortfall or surplus energy deliver are modeled. Finally, conclusions are addressed from a case study, using data from the pool-based EMar of the Iberian Peninsula.
\end{abstract}

Keywords: Bidding strategies - Wind power system - Stochastic linear programming $\cdot$ Day-ahead market

\section{Introduction}

A growing attention in ReEn sources has been followed over the last years due to its conversion into electric energy (EEn) free of pollutant emission and availability in all over the world [1]. E.U. countries are integrating ReEn into the electric grid (EGr) to fulfill Energy-2020 [2]. ReEn incentive has appeared and after 2012 the adoption by countries of one or even more incentives is a noteworthy fact [3]. Incentives include feed-in-tariff, guaranteed grid access, green certificates, investments incentives, tax credits and soft balancing (Bal) costs [4].

In restructured EMar, power producers (PPr) are entities owning power resources and taking part in the EMar with the objective of maximizing total profits. A PPr exploiting sources of WEn also face the uncertainty (Uncer) on the availability of these sources, meaning Uncer in complying with power contracts [5]. The closing of the EMar defines power trading and EPr. However, the remuneration depends on the conformity achieved on the level of the real deliver with the accepted value of the bid at the closing of the EMar. If no conformity is revealed, economic penalization for imbalances (Imb) is due to happen [6]. 
Uncers on sources of WEn can be accommodated to mitigate the final Uncer on the delivered energy (DEn). WEn cannot provide a continuous source of energy without Uncer due to the change of wind speed from one period to another [7]. Typically a WEn farm has more availability of WEn during the night and particular during the winter.

This paper is a research contribution to get the most out of WEn systems in order to conveniently accommodate BStr by the use of a CoA based in a SLPr approach.

\section{Relationship to Cyber-Physical Systems}

Power systems (PSy) face a shift from non-interactive, manually-controlled power grid (PGr) to the increased mixing of both cyber information and proper physical representations at all scales of the grid. A novel grid which features this cyber and physical grouping is the called Cyber-Physical Energy system (CyPES) [8]. ReEn reliance on weather conditions is a challenge in what regards integration into EGr [9]. A smart grid (SGr) provides flexibility needed to integrate ReEn. The SGr monitors energy flows, reducing cost and improving reliability [10]. Smart Systems (SSy) have been widely used in PSy [11]. The intelligent supervisory control and SCADA systems are pointed out as cyber-physical systems (CyPS) [12]. A CyPS is a combination of computation with physical processes [13]. CPS can be stated as a SSy, having software and hardware for sensing, monitoring, gathering, actuating, computing and controlling [14]. Cyber-Physical Intelligence (CyPI) is one topic to deal with SGrs, distributed generation and EMar [11]. The SGr needs CyPI to support tools for planning, EMar monitoring and risk management. Forecasting demand and EPr of EMar use Neural Network, Fuzzy Logic or both [15]. When big database about events is available, an ANN is a favorable option [15].

Multi-Agent System and Data Mining are considered for solving problems of EMar [15]. Intelligent systems are used for diagnosis and alarm processing at dispatch, at generation plant or substations. CyPES is estimated to improve the reliability, efficiency, flexibility, cost-effectiveness, and, very important, electric grid security [8] to avoid attacks [16].

\section{State of the Art}

Non-dispatchable ReEn sources, like WEn, are challenging for managing the bids in an EMar due to the Uncer in the unavailability of the ReEn, implying eventual FiPen for energy Imb [6]. So, the eventual deviations (Dev) and consequently penalty of Imb has to be considered. The technical literature presents methods for WEn BStr solved using different approaches. For example, in [17-21] PPr minimize the Dev losses through a portfolio approach, where the PPr can combine the WEn production with energy storage technologies so as to submit the optimal bids.

In [22], an approach is addressed so as to find out the OWBS, considering WEn production time series obtained from a Markov process. In [23] the Dutch EMar is analyzed, taking into account the robustness of the results obtained from point forecasts 
and probabilistic forecasts. In [17], the development of BStr is investigated for a WEn farm owner and a deterministic mixed-integer linear programming (MILP) approach for the optimal operation is proposed. In [17] is revealed an absence of treatment of WEn subjected to EMar price Uncer. In [24], linear programming is used for a WEn problem instead of mixed-integer nonlinear programming, concluding gains on robustness, simplicity and computational efficiency, implying adequacy to be integrated in SGrs in order to act in an enough small time processing, supporting decisions not only for helping bidding, but also for comparison in due time with eventual bilateral contracts. This linear programming used for the WEn problem is followed in this paper.

\section{Description of the Problem}

The wind energy traded (ETr) in a day-ahead electricity market (D-AEM) is subject to Uncers that must be considered in developing of offer strategies (OStr). The Uncers derive from the intermittence and volatility on the WEn and the EPr. These Uncers, if not adequately considered, can trigger losses of revenue (Rev) as a result of penalty incurred by the Imb. The Imb is set as a difference between the supplied energy ( $\mathrm{SpEn}$ ) and the offered energy (OfEn). If a surplus of SpEn in the PGr, then the Imb is positive (Posit); otherwise, the Imb is negative (Negat).

The system operator (SOp) minimizes the Imb value in a PGr using a methodology based on prices penalization for the Dev of the SpEn from the one offered by a producer and accepted in the closing D-AEM. In case of the Imb to be Negat, the SOp maintains the EPr for PPr with surplus of offers and pays a premium EPr produced above the offer. The EPr are as:

$$
\begin{gathered}
\vartheta_{x}^{+}=\vartheta_{x}^{D} \\
\vartheta_{x}^{-}=\max \left(\vartheta_{x}^{D}, \vartheta_{x}^{U P}\right)
\end{gathered}
$$

In (1) and (2), $\vartheta_{x}^{+}$and $\vartheta_{x}^{-}$, are used in the complementary EMar to the energy Dev, $\vartheta_{x}^{D}$ is the D-AEM price and $\vartheta_{x}^{U P}$ is the EPr for the energy that desires to be added to the system. In the case of the Imb to be Posit, the EPr are as:

$$
\begin{gathered}
\vartheta_{x}^{+}=\min \left(\vartheta_{x}^{D}, \vartheta_{x}^{D N}\right) \\
\vartheta_{x}^{-}=\vartheta_{x}^{D}
\end{gathered}
$$

In (3), $\vartheta_{x}^{D N}$ is the EPr of offers in exceeds. The Uncer in the available of ReEn source results in differences between the OfEn and the actual SpEn. The Rev $H_{x}$ for period $x$ is as:

$$
H_{x}=\vartheta_{x}^{D} I_{x}^{D}+P_{x}
$$


In (5), $I_{x}^{D}$ is the ETr in the D-AEM and $P_{x}$ is the Imb income derived from the Bal penalty.

The total Dev for period $x$ is as:

$$
\Theta_{x}=I_{x}-I_{x}^{D}
$$

Where $I_{x}$ is the actual power for period $x . P_{x}$ is set as:

$$
\begin{aligned}
& P_{x}=\vartheta_{x}^{+} \Theta_{x}, \Theta_{x} \geq 0 \\
& P_{x}=\vartheta_{x}^{-} \Theta_{x}, \Theta_{x}<0
\end{aligned}
$$

In (6), a Posit Dev happens (DevH) when the actual production is greater than the ETr in the D-AEM and a Negat DevH when the actual production lower than the ETr. In this manner, $\vartheta_{x}^{+}$is the EPr paid for surplus of production and $\vartheta_{x}^{-}$the EPr to be charged for the scarcity. Suppose that $\vartheta_{x}^{D}$ is not null and let:

$$
\begin{gathered}
r_{x}^{+}=\frac{\vartheta_{x}^{+}}{\vartheta_{x}^{D}}, r_{x}^{+} \leq 1, h_{x}^{+}=r_{x}^{+} \\
r_{x}^{-}=\frac{\vartheta_{x}^{-}}{\vartheta_{x}^{D}}, r_{x}^{-} \geq 1, h_{x}^{-}=r_{x}^{-}
\end{gathered}
$$

(9) and (10) are known respectively by the Posit and the Negat Imb EPr per unit of the closing EPr in the D-AEM. Substituting (9) and (10), respectively in (7) and (8), then:

$$
\begin{gathered}
P_{x}=\vartheta_{x}^{D} h_{x}^{+} \Theta_{x}, \Theta_{x} \geq 0 \\
P_{x}=\vartheta_{x}^{D} h_{x}^{-} \Theta_{x}, \Theta_{x}<0
\end{gathered}
$$

A producer that desires to rectify the energy Dev in a complementary EMar is subject to an opportunity cost, since D-AEMs have more competitive EPr.

Equation (5) can be rewritten to reveal the opportunity cost. Hence, in case of the energy Dev to be Posit, $\Theta_{x}>0$, the Rev is set as:

$$
H_{x}=\vartheta_{x}^{D} I_{x}^{D}+\vartheta_{x}^{D} h_{x}^{+} \Theta_{x}
$$

Using the total Dev stated in (6), the Rev is set as:

$$
H_{x}=\vartheta_{x}^{D} I_{x}^{D}-\vartheta_{x}^{D}\left(1-h_{x}^{+}\right) \Theta_{x}, \Theta_{x} \geq 0
$$

Also, in case of the energy Dev to be Negat, the Rev is set as: 


$$
H_{x}=\vartheta_{x}^{D} I_{x}^{D}+\vartheta_{x}^{D}\left(h_{x}^{-}-1\right) \Theta_{x}, \Theta_{x}<0
$$

Considering (14) and (15) can be expressed as:

$$
H_{x}=\vartheta_{x}^{D} I_{x}^{D}-C_{x}
$$

where:

$$
\begin{aligned}
& Z_{x}=\vartheta_{x}^{D}\left(1-h_{x}^{+}\right) \Theta_{x}, \Theta_{x} \geq 0 \\
& Z_{x}=-\vartheta_{x}^{D}\left(h_{x}^{-}-1\right) \Theta_{x}, \Theta_{x}<0
\end{aligned}
$$

In (16) $\vartheta_{x}^{D} I_{x}^{D}$ is the MRev obtained from ETr when Uncer is disregarded.

With Uncer a scenarios set $\Phi$ is accounted for WEn and Imbs. The scenario $\varphi$ is weighted with a probability of occurrence $\eta$. The $\eta$ is the same for $\varphi$.

The Rev over a time horizon is the objective function for the problem and is given as:

$$
\sum_{\varphi=1}^{N_{\Phi}} \sum_{x=1}^{N_{X}} \eta_{\varphi}\left(\vartheta_{x \varphi}^{D} I_{x}^{D}+\vartheta_{x \varphi}^{D} h_{x \varphi}^{+} \Theta_{x \varphi}^{+}-\vartheta_{x \varphi}^{D} h_{x \varphi}^{-} \Theta_{x \varphi}^{-}\right)
$$

In (19) $\vartheta_{x \varphi}^{D} I_{x}^{D}$ is the Rev from the accepted energy at the EPr of EMar closing, the other terms are the penalty for Imb.

The OWBS is given by the maximization of (19) subjected to the constraints as:

$$
\begin{gathered}
0 \leq I_{x}^{D} \leq I^{\max }, \forall x \\
\Theta_{x \varphi}=\left(I_{x \varphi}-I_{x}^{D}\right), \forall x, \forall \varphi \\
\Theta_{x \varphi}=\Theta_{x \varphi}^{+}-\Theta_{x \varphi}^{-}, \forall x, \forall \varphi \\
0 \leq \Theta_{x \varphi}^{+} \leq I_{x \varphi} d_{x}, \forall x, \forall \varphi
\end{gathered}
$$

In (20), the offers are limited by the technical range of operation, i.e., the installed maximum power in the WEn farm. In (21)-(23) is imposed that $\Theta_{t \varphi}^{+}=0$ when $\Theta_{t \varphi}^{+}$is Negat, $I_{x \varphi}<I_{x}^{D}$, and is imposed that $\Theta_{x \varphi}^{-}=0$ when $\Theta_{x \varphi}^{-}$is Negat, $I_{x}^{D}<I_{x \varphi}$.

When the system balance ( $\mathrm{SBal}$ ) is Negat, the producer is penalized for the deficit of energy generated (EnGen) below the ETr in the D-AEM, so the term $\vartheta_{x \varphi}^{D} h_{x \varphi}^{+} \Theta_{x \varphi}^{+}$is null and the term $\vartheta_{x \varphi}^{D} h_{x \varphi}^{-} \Theta_{x \varphi}^{-}$is subtracted from the Rev in the situation of no Dev, $\vartheta_{x \varphi}^{D} I_{x}^{D}$.

When the SBal is Posit, the producer is penalized for the EnGen above the ETr in the D-AEM, so the term $\vartheta_{x \varphi}^{D} h_{x \varphi}^{-} \Theta_{x \varphi}^{-}$is null and the term $\vartheta_{x \varphi}^{D} h_{x \varphi}^{+} \Theta_{x \varphi}^{+}$is added to the Rev in the situation of no Dev. 
Notice by the inequality in (3) that for the SBal Posit the added Rev is never greater than the Rev if no Posit Imb happens.

\section{Results}

The SLPr methodology is presented by a case study composed by two sets of data from the Iberian EMar [25].

The data set, i.e., price scenarios (PSc) includes 10 days of 2013-November and the other one 10 days of 2014-June shown in Fig. 1.
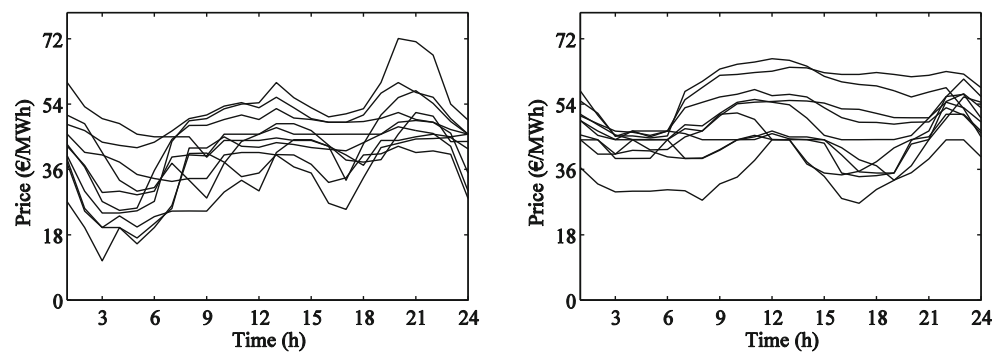

Fig. 1. PSc; (a) 2013-November, (b) 2014-June

Figure 1 shows hourly PSc at the closing of the D-AEM and each line is intended to represents a possible PSc.

The 2013 energy produced and 2014 energy produced are shown in Fig. 2.
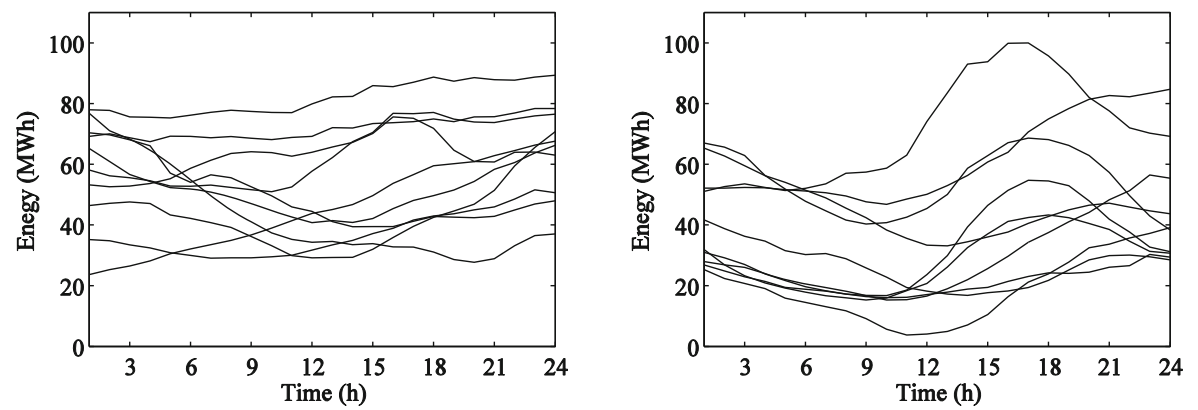

Fig. 2. WEn: energy (a) 2013-November, (b) 2014-June.

The EnGen is found through the total EnGen from the maximum power capacity of wind farm, $I^{\max }=120 \mathrm{MW}$.

The SOp matches the total EnGen to the system desires. This is reached by setting the price multipliers (PMul) $r_{t}^{+}$and $r_{t}^{-}$specified by (9) and (10). 
The $r_{t}^{+}$and $r_{t}^{-}$in 2013 are shown in Fig. 3 .
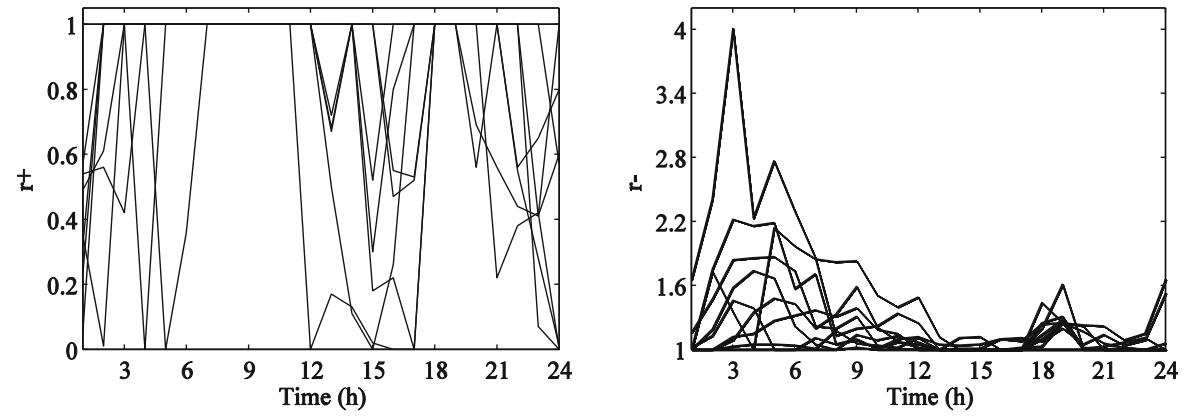

Fig. 3. Imb PMul 2013; (a) $r_{t}^{+}$, (b) $r_{t}^{-}$.

In 2014 the $r_{t}^{+}$and $r_{t}^{-}$are shown in Fig. 4.
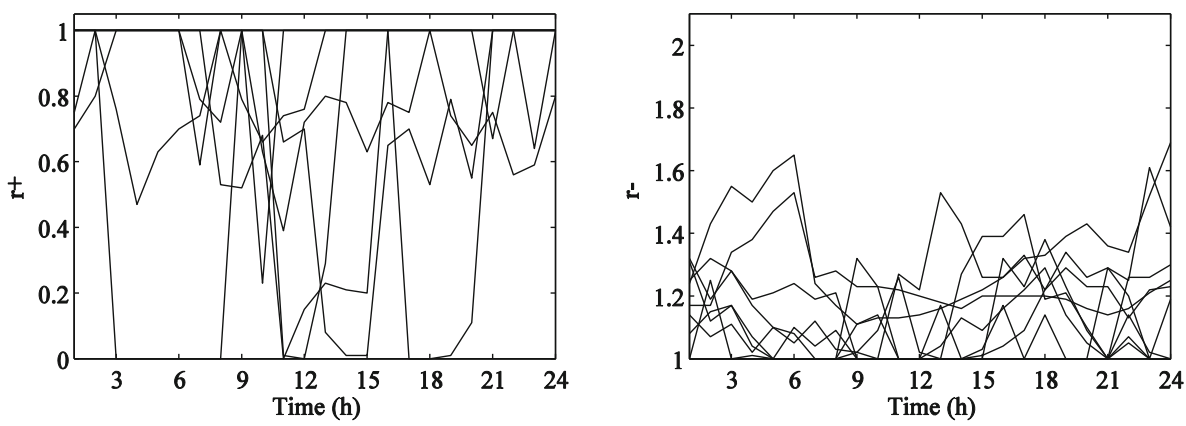

Fig. 4. Imb PMul 2014; (a) $r_{t}^{+}$, (b) $r_{t}^{-}$.

The optimal energy (OEn) offer that MRev estimated is determined by (19)-(23). The OEn offer and the estimated hourly revenue (EHRev) are shown in Figs. 5 and 6.

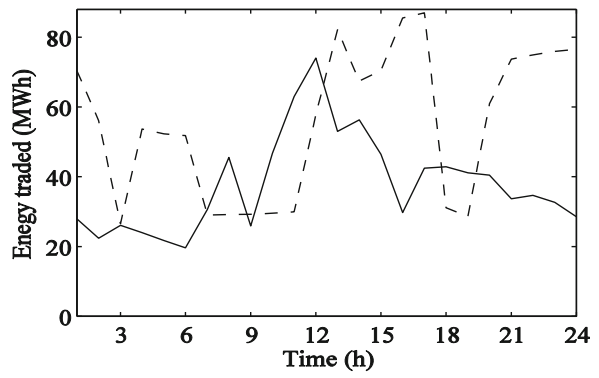

Fig. 5. ETr in D-AEM; 2013 dashed-line; 2014 solid line.

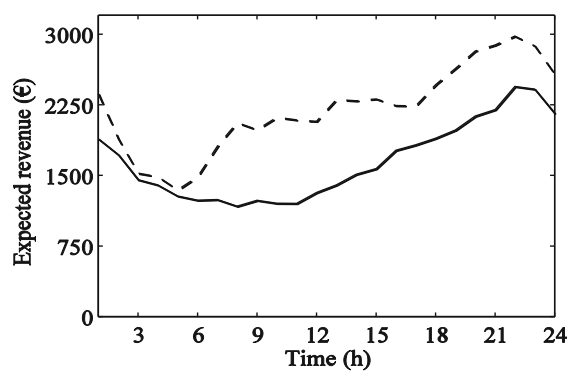

Fig. 6. EHRev; 2013 dashed-line; 2014 solid line 
For the $24 \mathrm{~h}$ the forecasted Rev in year 2013 is $52,861 €$ and in year $201439,656 €$.

The Rev Dev, considering that the 24 periods will be equal in $\varphi$, is shown in Fig. 7.

The Dev for the worst PSc (Fig. 7) is lower than the EHRev, implying that in the case of the condition over the day matches any one of the PSc, the outcome EHRev would be permanently positive.
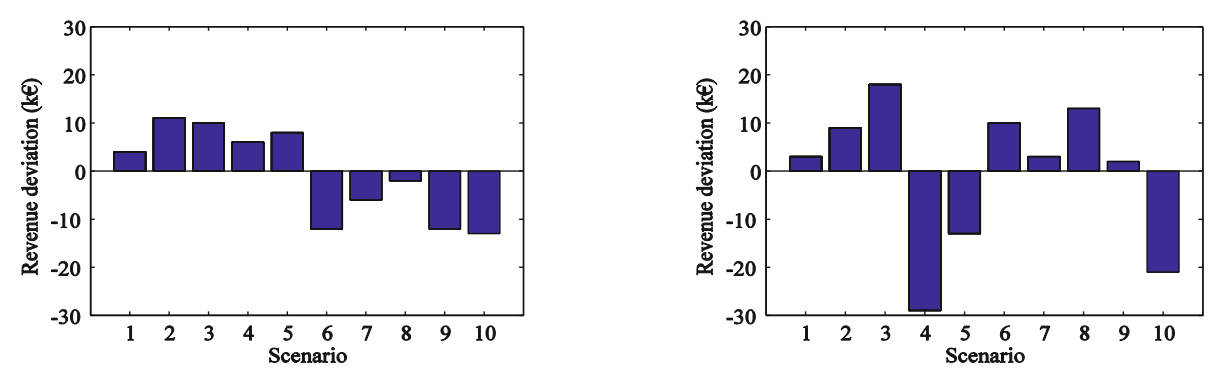

Fig. 7. Rev Dev; (a) 2013, (b) 2014.

\section{Conclusion}

A SLPr approach for solving the OStr of a wind PPr in a deregulated EMar is handled in this paper. The crutial result is the BStr for a wind PPr facing not only the wind power and EPr Uncers, but also the Imbs.

A SLPr approach is suitable to address OStr, in modeling via scenarios, if the computing resources needed are not excessive for use in a day basis.

The SLPr approach showed to be computationally acceptable, taking into consideration the number of PSc, and periods over the time horizon.

Additionality, the proposed approach is a useful tool for the PPr so as to be applied several times to reconfigured the bids according to short run changes of information.

Hence, the approach has adequacy to be integrated in SGrs in order to act in an enough small time processing, supporting decisions not only for helping bidding, but also for comparison with eventual bilateral contracts in due time.

Acknowledgments. The work presented in this paper is funded through Portuguese Funds by the Foundation for Science and Technology-FCT for project scope LAETA2015-2020, UID/EMS/50022/2013.

\section{References}

1. Tina, G., Gagliano, S., Raiti, S.: Hybrid solar/wind power system probabilistic modelling for long-term performance assessment. Sol. Energy 80, 578-588 (2006)

2. Energy 2020 - a strategy for competitive, sustainable and secure energy (2015). http:// europa.eu 
3. Ritzenhofen, I., Birge, J.R., Spinler, S.: The structural impact of renewable portfolio standards and feed-in-tariffs on electricity markets (2015). http://papers.ssrn.com/sol3/ papers.cfm?abstract_id=2418196

4. Wang, T., Gong, Y., Jiang, C.: A review on promoting share of renewable energy by green-trading mechanisms in power system. Renew. Sustain. Energy Rev. 40, 923-929 (2014)

5. Shrestha, G.B., Kokharel, B.K., Lie, T.T., Fleten, S.-E.: Medium term power planning with bilateral contracts. IEEE Trans. Power Syst. 20(2), 627-633 (2005)

6. Giannitrapani, A., Paoletti, S., Vicino, A., Zarrilli, D.: Bidding strategies for renewable energy generation with non stationary statistics. In: Proceedings of 19th International Federation of Automatic Control World Congress (2014)

7. Notton, G., Diaf, S., Stoyanov, L.: Hybrid photovoltaic/wind energy systems for remote locations. Energy Procedia 6, 666-677 (2011)

8. Al Faruque, M.A.: A model-based design of cyber-physical energy systems. In: Proceedings of 19th Asia and South Pacific Design Automation Conference (ASP-DAC), pp. 97-104 (2014)

9. Blaabjerg, F., Ionel, D.M.: Renewable energy devices and systems - state-of-the-art technology, research and development, challenges and future trends. Electr. Power Compon. Syst. 43(12), 1319-1328 (2015)

10. IEEE: Smart grid: reinventing the electric power system. In: IEEE Power and Energy Magazine for Electric Power Professionals. IEEE Power and Energy Society, USA (2011)

11. Ramos, C., Vale, Z., Faria, L.: Cyber-physical intelligence in the context of power systems. In: Kim, T.-h., Adeli, H., Slezak, D., Sandnes, F.E., Song, X., Chung, K.-i., Arnett, K. P. (eds.) FGIT 2011. LNCS, vol. 7105, pp. 19-29. Springer, Heidelberg (2011)

12. Faria, L, Silva, A., Ramos, C., Gomez, L., Vale, Z.: Intelligent behavior in a cyber-ambient training system for control center operators. In: Proceedings of 16th International Conference on Intelligent System Application to Power Systems, pp. 1-6 (2011)

13. Lee, E.A., Seshia, S.A.: Introduction to embedded systems - A cyber-physical systems approach. 2nd edn (2015). http://leeseshia.org/releases/LeeSeshia_DigitalV2_0.pdf

14. Foundations for Innovation in Cyber-Physical Systems - Workshop Summary Report. National Institute of Standards and Technology (2013)

15. Vale, Z.: Intelligent Power Systems. Wiley Encyclopedia of Computer Science and Engineering, Wiley, New York (2008)

16. Xie, L., Mo, Y., Sinopoli, B.: False data injection attacks in electricity markets. In: Proceedings of 1st IEEE Smart Grid Communications Conference (SmartGridComm), pp. 226-231 (2010)

17. García-González, J., Muela, R.M.R., Santos, L.M., González, A.M.: Stochastic joint optimization of wind generation and pumped-storage units in an electricity market. IEEE Trans. Power Syst. 23(2), 460-468 (2008)

18. García-González, J.: Hedging strategies for wind renewable generation in electricity markets. Proc. IEEE Power Energy Soc. Gen. Meet., Pittsburgh, USA (2008)

19. Angarita, J.M., Usaola, J.G.: Combining hydro-generation and wind energy biddings and operation on electricity spot markets. Electr. Power Syst. Res. 77(5), 393-400 (2007)

20. Bathurst, G.N., Strbac, G.: Value of combining energy storage and wind in short-term energy and balancing markets. Electr. Power Syst. Res. 67(1), 1-8 (2003)

21. Fabbri, A., San Roman, T.G., Abbad, J.R., Mendez Quezada, V.H.: Assessment of the cost associated with wind generation prediction errors in a liberalized electricity market. IEEE Trans. Power Syst. 20(3), 1440-1446 (2005)

22. Bathurst, G., Weatherill, J., Strbac, G.: Trading wind generation in short term energy markets. IEEE Trans. Power Syst. 17(3), 782-789 (2002) 
23. Pinson, P., Chevallier, C., Kariniotakis, G.: Trading wind generation from short-term probabilistic forecasts of wind power. IEEE Trans. Power Syst. 22(3), 1148-1156 (2007)

24. Morales, J., Conejo, A., Pérez-Ruiz, J.: Short-term trading for a wind power producer. IEEE Trans. Power Syst. 25(1), 554-564 (2010)

25. http://www.esios.ree.es/web-publica/ 